
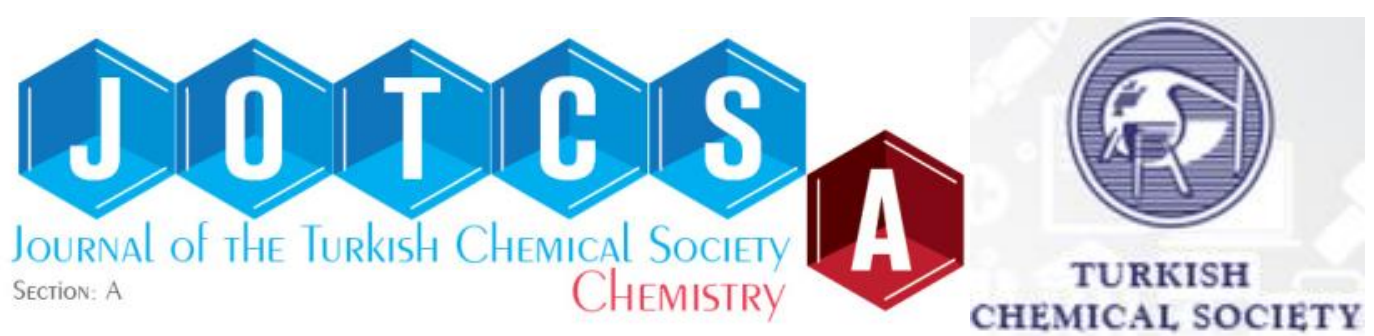

\title{
Artificial Intelligence Algorithms Inspired By Life Sciences
}

\author{
Hüseyin TURGUT*
}

Mehmet Akif Ersoy University, Vocational School of Technical Sciences, 15100, Burdur, Turkey

Abstract*: Nature and life include many mysterious events, behaviors and format within themselves. There is harmony between the environmental conditions, behavior and forms of all living organism. Computer science, especially data and information science, is based on the structure or behavior of living things in the creation of many artificial intelligence algorithms by examining this attitude of life. The rapid progress of the developing artificial intelligence and information technology has increased the data and hidden data in our lives many times and has tried to solve (1). Artificial intelligence has examined many areas or environments and has developed approaches based on it. Expert systems, artificial neural networks, genetic algorithms, inductive learning, explanation-based learning, similarity-based learning, common sense information processing, model based reasoning, model based reasoning, rational protection mechanism, distributed artificial intelligence, natural language processing, chaos theory, logic programming are the artificial intelligence algorithms used for these approaches (2). Among artificial intelligence algorithms; The ant colony algorithm imitates the behavior and direction of ants, and artificial neural networks imitates the behavior and functions of neurons in the nervous system and genetic algorithms imitates the theoretical form of genetic science $(3,4)$. Many algorithms such as these algorithms are based on the vital form and behavior of living things. The purpose of this review is the relations between the mentioned algorithms and the living science are examined.

Keywords: Artificial Intelligence, Life Sciences, Ant Colony, Artificial Neural Networks, Genetic Algorithm

$(*)$ This publication originated from the poster at the "International Chemistry \& Biology Conference '18 in Egypt" Congress.

Submitted: October 16, 2018. Accepted: October 22, 2018.

Cite this: Turgut $\mathrm{H}$. Artificial Intelligence Algorithms Inspired By Life Sciences. JOTCSA. 2018;5(3):1233-8.

DOI: http://dx.doi.org/10.18596/jotcsa.471300.

*Corresponding author. E-mail: hturgut@mehmetakif.edu.tr, web: hturgut.com.

\section{INTRODUCTION}

The process of making meaningful meaning, meaning meaningless imagery, is made possible by the command sequences called algorithms in computer science (5). This system, which runs the rules in order, is just an application. Learning involves stages such as understanding, perception and thinking. In general, machine learning is defined as the ability of computers to learn information and experiences about an event, to be able to decide on future events or to solve problems.

Machine learning is nowadays known as artificial intelligence. Artificial intelligence aims to develop computer processes that understand human thinking and bring out similarities; computer systems equipped with human intelligence-specific capacities such as 
information acquisition, perception, vision, thinking and decision-making.

Artificial intelligence is being developed by imitating human or natural intelligence. These imitations are inspired by the structural flow of many living or inanimate systems. This system, which is formed by working with many sciences, creates the greatest contribution in the identification and imitation process (6). Natural sciences are needed to improve the artificial intelligence and improve its performance. The nervous system, the genetic structure, the lives of animals and plants have created popular algorithms for artificial intelligence.

\section{ARTIFICIAL INTELLIGENCE ALGORITHM}

\section{Ant Colony Algorithm}

Natural Ants

The ants have the ability to find the shortest way to the source of food from their nest. They are making this move without hinting. They can get rid of obstacles and produce a new way (7). This function is shown in Figure 1.

The system used by the ants for the route finder is pheromone. Pheromone is a kind of chemical secretion that some animals use to influence other animals in their genus. The ants have some pheromones in motion. During the road finding process, the pheromone is preferred to the path with the least path. In this respect, the other ants who will come after themselves also help with the pheromones they leave. If an obstacle is encountered or can not be maintained, the ant will select a path with equal probability and create a new path. This ventricle is called the leading ant. If it reaches food, it will return to the house using the same way. This route will be the proper route because the route it follows will take more pheromones. The new shortest path will take place thanks to the fact that later ants choose this new path (7).

\section{Ant Colony Algorithm - Virtual Ants}

Researchers produce a new algorithm for solving problems by examining the behaviors of ants. The generated algorithm imitates the progress of these ants in the nature leaving traces. The structure formed by this method is called "virtual ant". Computer Science shows that most problems simulated by creating "virtual tinkering" can be solved more easily (8). The most popular problem solved using this algorithm is the traveler seller problem. The first study was conducted in 1991 by Dorigo et al (7).

Since it is inspired by the ant colony, the system is called the "ant system"; The algorithm is called the "ant colonies algorithm".

The algorithm is like this:

1. Initial pheromone values are determined.

2. The ants are placed randomly at each knot.

3. Each ant completes the round by selecting the next address based on the local search probability given in the equation.

4. The distance traveled by each ant is calculated and the local pheromone is updated.

5. The best solution is calculated and used in global pheromone renewal.

6. Go to Step 2 until the maximum number of iterations or qualification criteria is met.

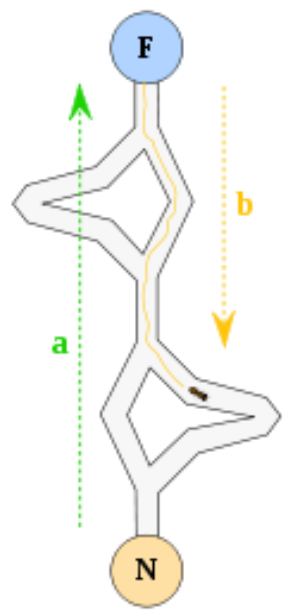

1

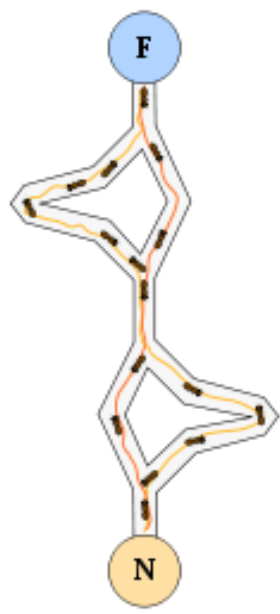

2

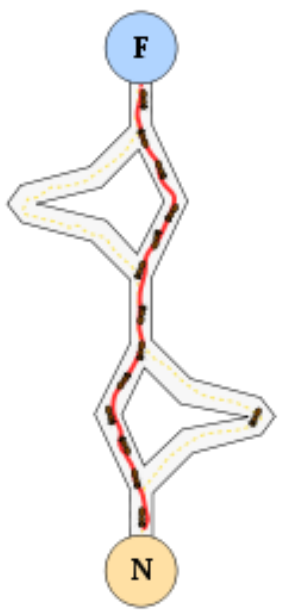

3

Figure 1: Simulation of ant colony algorithms (9)

ANN algorithms are often used to produce the nearest formula in problem solving. It can be 
used to determine the route of distribution of a cargo company or to reach the closest results of search engines (9).

\section{Genetic Algorithms}

Genetic algorithms are a search and optimization method that works in a manner similar to the evolutionary process observed in nature. In the complex multi-dimensional search space, the best solution is holistic according to the principle of survival of the best.

The basic principles of genetic algorithms were first proposed by John Holland at the University of Michigan. Holland, in 1975, assembled his work in his book "Adaptation in Natural and Artificial Systems". First, Holland used evolutionary laws for optimization problems in genetic algorithms $(10,11)$. Genetic algorithms generate a set of solutions consisting of different solutions rather than producing a single solution to the problems. The solutions in the solution set are completely independent of each other. Each is a vector on multidimensional space. Genetic algorithms simulate the evolutionary process in computer environment for solving problems. This cluster, representing many possible solutions to the problem, is called "population" in the genetic algorithm terminology. A population consists of a number sequence called a vector, chromosome, or individual. Each element within an individual is called a "gene". The most important factor in deciding the success of genetic algorithms in solving the problem is the representation of the individuals representing the solution of the problem. There is a "conformity function" that decides whether each individual in the population will be a solution to the problem. According to the return value from the fitness function, individuals with high values are given the opportunity to multiply with other influential individuals. These individuals produce new individuals called "children" at the end of the crossing process. The child carries the characteristics of parents (mother, father) who bring themselves to the festival. Since individuals with low fitness values will be chosen less, these individuals are excluded from the population after a while. The new population is formed by the gathering of highfit individuals in the previous population. Thus, through many generations, good traits are propagated in the population and are combined with other good traits through genetic processes. The greater the number of individuals with higher fitness values come together to create new individuals, the better the working space is obtained within the search space $(10,11)$.

For the best solution;
- The representation of the individual must be done correctly,

- The conformity function must be established effectively,

- The right genetic operators should be chosen.

Genetic algorithms are effective and useful only in areas where search space is large and complicated, resolution is difficult in the search space limited by the available information, where the problem can not be expressed by a specific mathematical model, and where the desired result is not obtained from conventional optimization methods.

\section{Difference from other methods}

1-Genetic algorithms search for solutions of problems by their codes, not by the values of parameters. The solution can be produced as long as the parameters can be coded. For this reason, genetic algorithms do not know what they are doing, they know how to do it.

2-Genetic algorithms begin with a set of points, not a single point of search. For this reason, they are often not trapped in the local best solution.

3-Genetic algorithms use the value of the fitness function instead of the derivative. Use of this value also does not require the use of auxiliary information.

4-Genetic algorithms use probabilistic rules, not necessity rules

Genetic algorithms are used in studies with function optimization. This artificial intelligence algorithm is more effective in terms of difficulty, continuity and noise from traditional optimization techniques (12).

\section{Artificial Neural Networks}

A biological nerve cell consists of four parts in total. These are the trunk, axon, numerous dendrites and synapses. Dendrites transmit the incoming signals to the nucleus. The nuclei collect the signals from the dendrites and transmit them to the axon. These collected signals are processed by axon and sent to synapses. Synapses also transmit newly produced signals to other nerve cells.

Artificial Neural Cell

Artificial Neural Networks (ANN) are computer systems developed with the intention of automatically generating the ability to derive new information, learn new information, and discover new ones through learning from the characteristics of the human brain without any help. It is difficult or impossible to realize these capabilities with traditional programming methods. For this reason, artificial neural networks can be described as a computer science dealing with adaptive information processing developed for very difficult or impossible events (6). 
Entrances from the outside or other cells are connected to the cell by means of weights. The net input is calculated together with the summation function. The net output is calculated by passing the pure input through the activation function. This process is also equal to the exit of the cell (13).

Elements of Artificial Neural Cell

- Inputs: Information from the outside world or from another cell to the artificial neural networks.

- Weights: Represents the numerical value of the connections between cells. It shows the value of information coming to a cell and its effect on the cell.

- Aggregation Function: Calculates the net input of that cell by multiplying the input from the cell by weights.

- Activation Function: It processes the net entry into the cell and allows the cell to determine whether it will produce a response to this input.



- Outputs: Output values determined by the activation functions. The output produced can either be sent to the outside world, to another cell or to itself as input.

\section{ANN Structure}

Input Layer: The layer from which the entrances come from the outside world. In this layer, there are as many cells as there are inputs, and the inputs are transmitted to the hidden layer without any processing.

Hidden Layers: Transfers the information from the entry layer to the next layer. The number of hidden layers and the number of cells in the hidden layer may vary in each network. Cell numbers in hidden layers are independent of input and output numbers.

Output Layer: Processes data from hidden layer. The output generated according to the input to the entrance layer transfers to the outside world. The number of cells in the output layer can be more than one. Each output cell has one output. Each cell is dependent on all the cells in the previous layer.

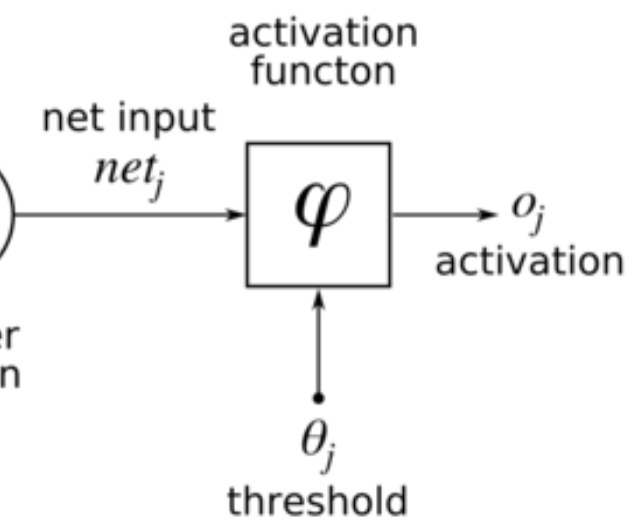

Figure 2. Structure of ANN algorithm (6)

\section{Properties of ANN}

- They have the ability to self-organize and learn.

- They can work with missing data.

- Has fault tolerance.

- They can process indefinite and incomplete information.

- ANN does not exhibit sudden deterioration.

- They can only work with numerical information.

- ANN is designed to solve problems that are difficult to solve by known solutions.

\section{Advantages of ANN}

- ANN is successful in situations where there is no definite mathematical model or algorithm to solve the problem.
- Successful results are found in situations with a large number of exceptional and abnormal data.

- Has the ability to adapt.

- Information is stored entirely on the network.

- Produce information about previously unseen samples.

\section{Disadvantages of ANN}

- 'Black Box' can not explain the final result.

- There is no specific rule for determining proper network structure.

- There is no specific rule for setting network parameter values.

- There is no general rule in selecting training samples.

- Displaying the problem to the network is an important problem. 
- There is no specific method of when network training should be finished.

\section{DISCUSSION \& CONCLUSIONS}

Computer science has created algorithms called programming, primarily to speed up people's work and save on labor power. These algorithms mimic only the work done. For example, paper work done by an officer in daily life is now made faster and more economically thanks to the computer. However, artificial intelligence, in addition to imitating the work of human beings, allows people to make decisions in their work and reach the results without doing business.

Computer technologies not only accelerate work but also offer solutions. Social life and scientific processes are accelerated by using Computer Programming. However, artificial intelligence technology can imitate the solutions of nature. Life and biology have been imitated in the creation of many artificial intelligence algorithms. The ants, neurons, genetic structure are examples of these.

\section{REFERENCES}

1. Fayyad U, Stolorz P. Data mining and KDD: Promise and challenges. Future generation computer systems. 1997;13(23):99-115.

2. Baykal N, Beyan T. Bulanık mantık: uzman sistemler ve denetleyiciler. Bıçaklar Kitabevi; 2004.

3. Rosenblatt F. The perceptron: a probabilistic model for information storage and organization in the brain. Psychological review. 1958;65(6):386.

4. Holland JH. Genetic algorithms. Scientific american. 1992;267(1):66-73.

5. Moschovakis YN. What is an algorithm? Içinde: Mathematics unlimited-2001 and beyond. Springer; 2001. s. 919-936.

6. Turgut $H$. Veri madenciliği süreci kullanılarak alzheimer hastalığı teşhisine yönelik bir uygulama [Master Thesis]. Süleyman Demirel Üniversitesi Fen Bilimleri Enstitüsü; 2012

7. Dorigo M, Maniezzo V, Colorni A. Dipartimento Di Elettronica-Politecnico Di Milano. 1991;

8. Parpinelli RS, Lopes HS, Freitas AA. An ant colony algorithm for classification rule discovery. Içinde: Data mining: A heuristic approach. IGI Global; 2002. s. 191-208.

9. Maniezzo V, Gambardella L, Luigi FD. New Optimization Techniques in Engineering. Içinde: An ANTS Heuristic for the Long-Term Car Pooling Problem. Springer Berlin, Heidelberg; 2004. s. 411-430.

10. Gen M, Cheng R, Oren SS. Network design techniques using adapted genetic algorithms. Içinde: Evolutionary Design and Manufacture. Springer; 2000. s. 107-120.

11. Beasley D, Bull DR, Martin RR. An overview of genetic algorithms: Part 1, fundamentals. University computing. 1993;15(2):56-69.

12. Beasley D, Bull DR, Martin RR. A sequential niche technique for multimodal function optimization. Evolutionary computation. 1993;1(2):101-125.

13. Wang S-C. Interdisciplinary computing in Java programming. C. 743. Springer Science \& Business Media; 2012. 
Turgut H. JOTCSA. 2018; 5(3): 1233-1238.

REVIEW ARTICLE 